\title{
Preference of water-deprived rats for stimulation of the lateral hypothalamus rather than water ${ }^{1,2}$
}

\author{
C. W. MORGAN AND G. J. MOGEHSON
}

THE UNIVERSITY OF WESTERN ONTARIO, LONDON, CANADA

Rats with electrodes implanted in the lateral hypothalamus had a choice of lever pressing for water or brain stimulation. Lever preference varied depending on stimulation intensity and degree of water deprivation. With lower current levels the animals responded normally to the lack of water and selected the water lever. However, when higher intensities were used they chose the brain stimulation lever and did not satisfy their water requirements even after $71 \mathrm{hr}$. of water deprivation.

The lateral hypothalamus is a critical focus of the control systems for water and food intake (Hamilton, 1965; Stevenson, 1965). Nevertheless, rats will perform instrumental responses for electrical stimulation of this region even if it requires that they ignore food after several days of food deprivation (Routtenberg \& Lindy, 1965; Spies, 1965) or that they tolerate painful grid shock (Olds, 1958). The present study was designed to determine if rats also prefer electrical stimulation of the lateral hypothalamus to the ingestion of water after a period of water deprivation.

Method

Eleven male Wistar rats, anesthetized with sodium pentobarbital, were implanted with bi-polar electrodes (Plastic Products Company, Roanoke, Virginia, MS 303.018") in the lateral hypothalamus. The electrodes were cemented permanently to the skull with cranioplastic cement. The stereotaxic coordinates for the electrodes were according to DeGroot: $A=5.0$ to 5.5, $L=1.75, v=-2.0$ to -2.5 . Beginning about a week later the animals were trained in a Skinner box to selfstimulate. Each lever press delivered a $0.2 \mathrm{sec}$. train from a 60 cycle ac constant-current stimulator. The animals were next placed on a 23-hr. water deprivation schedule and trained to press a lever for water reward which consisted of $.05 \mathrm{ml}$ of water being delivered into a small dish near the lever after each lever press. When this training schedule was completed the animals were tested in a chamber ( 20 in. by 12 in.) with two levers (Lehigh Valley Electronics, Model 1535) inserted 6 in. apart in one of the longer walls. Pressing one lever activated the stimulator, the other activated the liquid pump (Davis Scientific Instruments, Model LR131) which delivered water. For one week a divider was placed between the two levers and the animals were given an opportunity to press $15 \mathrm{~min}$. for water reward and $15 \mathrm{~min}$. for brain stimulation each day. During the next three weeks the animals were tested with the divider removed so that they had access to both levers. The response to currents varying from 1 to $50 \mu \mathrm{A}$ was determined. A current just below the threshold at which self-stimulation began (mean 7.75 $\mu \mathrm{A}$ peak current) was called level I and the current at which self-stimulation did not increase any further (mean $29.50 \mu \mathrm{A}$ ) was called level $\mathrm{V}$. Current levels II, III and IV were determined for each rat by taking equal intervals from level I to level $\mathrm{V}$.

The preference of the animals for self-stimulation or water was tested at each of the five levels of stimulation current after each of four periods of water deprivation $(0,23,47$, and $71 \mathrm{hr}$.). The test period lasted 15 min. and each animal was tested twice under each of the conditions. The temporal order of these conditions was varied among rats. The number of presses on each lever for the 20 conditions was recorded. Analyses of variance for repeated measures were made on both sets of scores and, as one response rate was the complement of the other it was found that both analyses supported the same conclusions. Since the study was concerned with the effect on water intake of the competitive behavior of self-stimulation, only the analysis of the presses on the water lever is presented.

The exact site of the electrode tips was determined histologically by examination of sections of the hypothalamus cut at 15 microns and stained with cresyl violet.

Results

At the lowest current level the responses of the animals were predominantly for water reward after 23, 47 and $71 \mathrm{hr}$. water deprivation (Fig. 1). As the current level for self-stimulation increased from level I to level $\mathrm{V}$ the presses on the water lever decreased

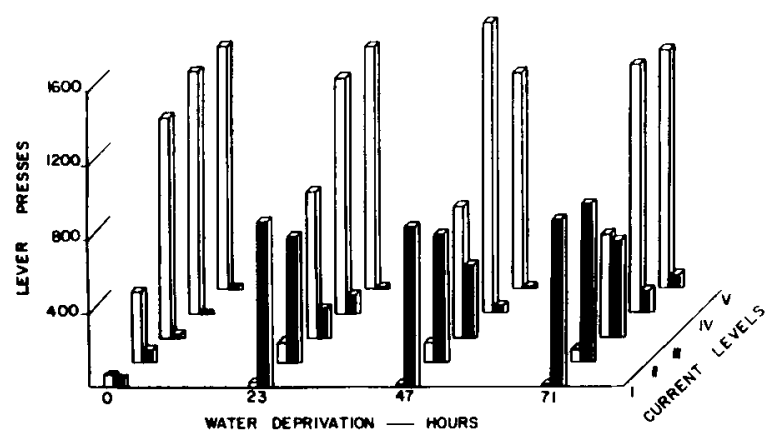

Fig. 1. Mean lever presses for water (black columns) and for electrical stimulation of the lateral hypothalamus (white columns) during 15-min. sessions. 
and the presses on the stimulation lever increased until at the highest current level the responses were mainly for brain stimulation.

The current level was a highly significant variable $(\mathrm{F}=83.7, \mathrm{p}<.001)$ as was the duration of water deprivation $(F=73.5, p<.001)$. There was also a significant interaction ( $F=22.7, p<.001)$ as the effect of increasing water deprivation was very marked at the lower current levels but virtually disappeared at the higher levels.

Electrodes were all found to terminate in the medial aspect of the medial forebrain bundle between anterior 5.0 and anterior 5.5 (De Groot, 1959). These placements are more anterior than those reported by Routtenberg \& Lindy (1965) or by Spies (1965). In three of the animals the electrodes were in the region dorsolateral to the fornix in which electrical stimulation elicites drinking behavior (Mogenson \& Stevenson, 1966).

\section{Discussion}

In the rat the lateral hypothalamus is one of the most effective sites at which to elicit self-stimulation and the only locus from which drinking has yet been induced by electrical stimulation. At higher current levels of short duration, which presumably activate the reward system more effectively, the animals self-stimulated in preference to drinking water even after $71 \mathrm{hr}$. of water deprivation. This neglect of the normal homeostatic response is similar to that seen in the fooddeprived animal when feeding is placed in competition with self-stimulation of the lateral hypothalamus (Routtenberg \& Lindy, 1965; Spies, 1965).

It has been suggested that self-stimulation of the lateral hypothalamus serves as a food equivalent (Routtenberg \& Lindy, 1965). In three of the 11 rats used in the present study electrical stimulation of the lateral hypothalamus so:cited drinking behavior (Mogenson \& Stevenson, 1966). These rats, like the other eight animals, pressed for self-stimulation at the higher current levels even though they had been deprived of water for $71 \mathrm{hr}$., at which time they did not make a single lever press for water but pressed for brain stimulation 600 , 2345 and 2049 times, respectively. Stimulation of the lateral hypothalamus may act centrally as an equivalent for water.

The rat does not prefer self-stimulation of all regions of the reward system to the natural responses resulting from food and water deprivation. Animals with the electrodes in the septal region preferred food to self-stimulation after food-deprivation (Routtenberg \& Lindy, 1965; Spies, 1965) and water to self-stimulation after water deprivation (Falk, 1961). The preference for self-stimulation in the lateral hypothalamus over the natural response to a deprivation depends upon the duration of the individual stimulus as well as its intensity. Mogenson \& Stevenson (1966) have reported that with durations of stimulation of more than $1 \mathrm{sec}$. the animal will drink in response to each stimulus train and it will continue to self-stimulate and drink to the point of continuing diuresis. Explanation of this contrast between the intensity and duration of the individual stimulus on the preference of the rat for self-stimulation in the lateral hypothalamus remains to be determined.

\section{References}

De Groot. J. The rat fore br ain in stereotaxic coordinates. Amsterdam: N-V Noord-Hollandsche Vitgevers Moatsckopy, 1959.

Falk, J. L. Septal stimulation as a reinforcer of, and an alternative to, consummatory behavior. J. exp. Anal. Behav., 1961, 4, 213217.

Hamilton, C. L. Control of food intake. In W. S. Yamamoto \& J. R. Brobeck (Eds.), Physiological controls and regulations. Philadelphia: Saunders, 1965. Pp. 274-294.

Mogenson, G. J., \& Stevenson, J. A. F. Drinking and self-stimulation with electrical stimulation of the lateral hypothalamus. Physiol. \& Behavior, 1966, 1, 251-254.

Olds, J. Self-stimulation of the brain. Science, 1958, 127, 315-324.

Routtenberg, A., \& Lindy, J. Effects of availability of rewarding septal and hypothalamic stimulation on bar pressing for food under conditions of deprivation. J. comp. physiol. Psychol., $1965,60,158-161$

Spies, G. Food versus intracranial self-stimulation reinforcement in food-deprived rats. J. comp. physiol. Psychol., 1965, 60, 153-157.

Stevenson, J. A. F. Control of water exchange: regulation of content and concentration of water in the body. In W. S. Yamamoto \& J. R. Brobeck (Eds.), Physiological controls and regulations. Philadelphia: Saunders, 1965, Pp. 253-273.

\section{Notes}

1. Supported by the National Research Council of Canada and the Medical Research Council of Canada.

2. The interest and critical suggestions of Dr. J. A. F. Stevenson are gratefully acknowledged. 